\title{
POINTWISE ESTIMATES OF THE SIZE OF CHARACTERS OF COMPACT LIE GROUPS
}

\author{
KATHRYN E. HARE, DAVID C. WILSON and WAI LING YEE
}

(Received 17 April 1999; revised 15 February 2000)

Communicated by A. H. Dooley

\begin{abstract}
Pointwise bounds for characters of representations of the classical, compact, connected, simple Lie groups are obtained which allow us to study the singularity of central measures. For example, we find the minimal integer $k$ such that any continuous orbital measure convolved with itself $k$ times belongs to $L^{2}$. We also prove that if $k=\operatorname{rank} G$ then $\mu^{2 k} \in L^{1}$ for all central, continuous measures $\mu$. This improves upon the known classical result which required the exponent to be the dimension of the group $G$.
\end{abstract}

2000 Mathematics subject classification: primary 43A80; secondary 22E46, 43A65.

Keywords and phrases: central measures, corfpact Lie group, characters.

\section{Introduction}

In this paper sharp, pointwise bounds for characters of representations of the classical, compact, connected, simple Lie groups are obtained. Our prime motivation is to use these estimates to study the singularity of central, continuous measures.

In [8] Ragozin proved the striking fact that if $G$ was such a group and $\mu$ was a central, continuous measure on $G$, then $\mu^{\operatorname{dim} G} \in L^{1}(G)$ (the product here is convolution). This implies, in particular, that if $g$ is not in the centre of $G$, then $\operatorname{Tr} \lambda(g) / \operatorname{deg} \lambda \rightarrow 0$ as the degree of the representation $\lambda$ tends to infinity [11]. Ragozin's result was improved by one of the authors in [2] where it was shown that if $g$ does not belong to the centre

The authors would like to thank F. Zorzitto for helpful conversations.

The research of the first author and the third author was partially supported by NSERC.

The hospitality of the University of Waterloo is gratefully acknowledged by the second author.

(C) 2000 Australian Mathematical Society 0263-6115/2000\$A2.00+0.00 
of $G$, then

$$
\left|\frac{\operatorname{Tr} \lambda(g)}{\operatorname{deg} \lambda}\right| \leq c(g)(\operatorname{deg} \lambda)^{-2 /(\operatorname{dim} G-\operatorname{rank} G)} .
$$

A consequence of this bound on the trace function is that if $k>\operatorname{dim} G / 2$ and $\mu$ is a continuous orbital measure, then $\mu^{k} \in L^{2}(G)$, while if $\mu$ is any central, continuous measure then $\mu^{k} \in L^{1}(G)$.

In this paper we improve these results, obtaining the following theorem for classical Lie groups of rank $n$.

THEOREM 1.1. Let $G$ be a compact, connected, simple Lie group of type $A_{n}, B_{n}, C_{n}$ or $D_{n}$. For every $g$ not in the centre of $G$ there is a constant $c(g)$ such that

$$
\left|\frac{\operatorname{Tr} \lambda(g)}{\operatorname{deg} \lambda}\right| \leq c(g)(\operatorname{deg} \lambda)^{-s}
$$

for all representations $\lambda$ if and only if

$$
s \leq \begin{cases}1 /(n-1) & \text { if } G \text { is type } A_{n-1} \text { or } D_{n} ; \\ 1 /(2 n-1) & \text { if } \bar{G} \text { is type } B_{n} \\ 2 /(2 n-1) & \text { if } G \text { is type } C_{n}, n \neq 3 \\ 1 / 3 & \text { if } G \text { is type } C_{3} .\end{cases}
$$

(In contrast, $\operatorname{dim} G-\operatorname{rank} G=O\left(n^{2}\right)$.)

From Theorem 1.1 we are able to show that if $G$ is type $A_{n-1}, C_{n}$ for $n \neq 3$ or $D_{n}$, and $\mu$ is any continuous, orbital measure, then $\mu^{k}$ belongs to $L^{2}(G)$ if and only if $k \geq \operatorname{rank} G=n$. Furthermore, if $\mu$ is any continuous, central measure, then $\mu^{n}$ belongs to $L^{1}(G)$. For type $B_{n}$ the condition is $k \geq 2 n$.

Key to proving Theorem 1.1 is to understand the structural properties of maximal subroot systems. These are discussed in Section 2 . In Section 3 we use these properties and computational arguments based on the Weyl character formula to establish the specified pointwise upper bounds on the trace function. Examples are found in Section 4 which prove these upper bounds are best possible. Applications to the study of the singularity of central measures can be found in Section 5 .

\section{Notation and structural properties of subroot systems}

2.1. Notation Let $G$ be a compact, connected, simple, non-exceptional Lie group of rank $n$. Let $Z(G)$ denote its centre and $W$ be its Weyl group. Denote by $e_{1}, \ldots, e_{m}$ 
the usual unit vectors in $\mathbb{R}^{m}$, where $m=n+1$ in type $A_{n}$ and $m=n$ otherwise. We take a maximal torus $T$ with $\Phi$ the set of roots for $(G, T)$ described below.

Type Root system $\Phi$

$A_{n} \quad\left\{e_{i}-e_{j}: 1 \leq i \neq j \leq n+1\right\}$

$B_{n} \quad\left\{ \pm e_{i}, \pm\left(e_{i} \pm e_{j}\right): 1 \leq i \neq j \leq n\right\}$

$C_{n} \quad\left\{ \pm 2 e_{i}, \pm\left(e_{i} \pm e_{j}\right): 1 \leq i \neq j \leq n\right\}$

$D_{n} \quad\left\{ \pm\left(e_{i} \pm e_{j}\right): 1 \leq i \neq j \leq n\right\}$
Base $\Delta=\left\{\alpha_{j}: j=1, \ldots, n\right\}$

$\alpha_{j}=e_{j}-e_{j+1}$

$\alpha_{j}=e_{j}-e_{j+1}$ for $j \neq n$

$\alpha_{n}=e_{n}$

$\alpha_{j}=e_{j}-e_{j+1}$ for $j \neq n$

$\alpha_{n}=2 e_{n}$

$\alpha_{j}=e_{j}-e_{j+1}$ for $j \neq n$

$\alpha_{n}=e_{n-1}+e_{n}$

The set of positive roots associated with the base of simple roots $\Delta$ is denoted by $\Phi^{+}$, the fundamental dominant weights relative to $\Delta$ are denoted by $\lambda_{1}, \ldots, \lambda_{n}$, and $\Lambda^{+}$ is the set of all dominant weights. The set $\Lambda^{+}$is in a 1-1 correspondence with $\widehat{G}$; $\sigma_{\lambda} \in \widehat{G}$ is indexed by its highest weight $\lambda \in \Lambda^{+}$. The degree of $\sigma_{\lambda}$ is denoted by $d_{\lambda}$. The weights of $\lambda \in \Lambda^{+}$are given by

$$
\Pi(\lambda)=\{\mu \in \Lambda: w(\mu)<\lambda \text { for all } w \in W\},
$$

where $\mu<\lambda$ means $\lambda-\mu$ is a non-negative integral sum of positive roots. We set $\rho=\sum_{j=1}^{n} \lambda_{j}$. According to the Weyl dimension formula [13] the degree of $\lambda$ is given by

$$
\prod_{\alpha \in \Phi^{+}}(\hat{\rho}+\lambda, \alpha) /(\rho, \alpha)
$$

For general facts about root systems we refer the reader to [5].

Given $g \in T$ we let $\Phi(g)=\{\alpha \in \Phi: \alpha(g) \in 2 \pi \mathbb{Z}\}$ and let $\Phi^{+}(g)=\Phi(g) \bigcap \Phi^{+}$. It is easily seen that $\Phi(g)$ is a subroot system of $\Phi$ and that $\Phi^{+}(g)$ is a complete set of positive roots of this subroot system. It is known that $\Phi(g)=\Phi$ if and only if $g \in Z(G)$ [1, page 189]. When $\Phi(g)$ is empty $g$ is called a regular element of $G$.

For $g$ in the torus, the Weyl character formula ([13]) states

$$
\operatorname{Tr} \lambda(g)=\frac{e^{i \rho(g)} \sum_{w \in W} \operatorname{det} w \exp i(\rho+\lambda, w(g))}{\prod_{\alpha \in \Phi^{+}}\left(e^{i \alpha(g)}-1\right)} .
$$

This determines $\operatorname{Tr} \lambda$ on $G$ as characters are class functions.

When $g \in Z(G)$ an application of Schur's lemma shows that $|\operatorname{Tr} \lambda(g)|=d_{\lambda}$, hence the interest is when $g \notin Z(G)$. It was shown in [2] how one can evaluate the Weyl character formula (by considering suitable directional derivatives if $\Phi^{+}(g)$ is not empty) to obtain

$$
\frac{|\operatorname{Tr} \lambda(g)|}{d_{\lambda}}=c(g) \frac{\left|\sum_{w \in W} \operatorname{det} w \prod_{\alpha \in \Phi^{+}(g)}(\rho+\lambda, w(\alpha)) \exp i(\rho+\lambda, w(g))\right|}{\prod_{\alpha \in \Phi^{+}}(\rho+\lambda, \alpha)} .
$$




$$
A_{n}, n \geq 2
$$

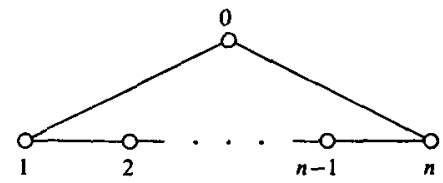

$$
B_{n}, n \geq 3
$$

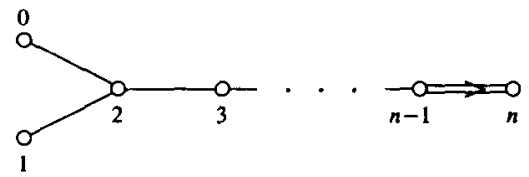

$C_{n}, n \geq 2$

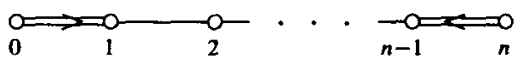

$D_{n}, n \geq 4$

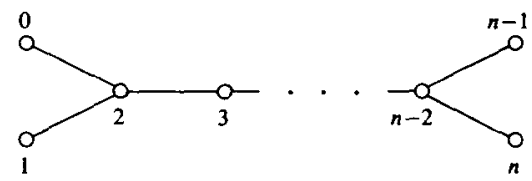

FIGURE 1. Extended Dynkin diagrams.

Consequently,

$$
\frac{|\operatorname{Tr} \lambda(g)|}{d_{\lambda}} \leq c(g) \frac{\sum_{w \in W}\left|\prod_{\alpha \in \Phi^{+}(g)}(\rho+\lambda, w(\alpha))\right|}{\prod_{\alpha \in \Phi^{+}}(\rho+\lambda, \alpha)} .
$$

Thus in order to find pointwise bounds on the trace functions off the centre of $G$ it is useful to understand the structures of the subroot systems properly contained in $\Phi$. It clearly suffices to analyze those subroot systems which are maximal in the sense that there is no other proper subroot system containing it. These subroot systems are always associated with regular subalgebras, (although not always of maximal rank) and hence their diagrams are subdiagrams of the extended diagram of the original root system (see Figure 1). Note that the additional vertex, labelled 0 , is identified with the highest root $\alpha_{0}$.) Once all these subdiagrams have been identified we can determine all possible sets of positive roots associated with maximal subroot systems by considering Weyl conjugates of the bases corresponding to the subdiagrams.

We illustrate how to do this to find the positive roots of all maximal subroot systems for type $B_{n}$. The other types are summarized below.

2.2. Maximal subroot systems of type $B_{n}$ Consider the extended diagram of Figure 2. Notice that if vertex 0 or 1 is removed the remaining subgraph is still type $B_{n}$ and thus is not proper. If vertex 2 is removed we are left with type $A_{1} \times A_{1} \times B_{n-2}$. 
extended $B_{n}$

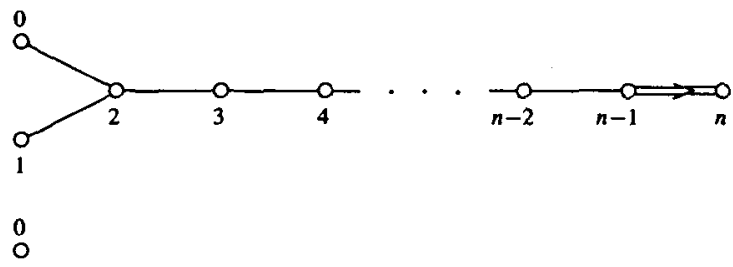

$D_{2} \times B_{n-2}$

0
1

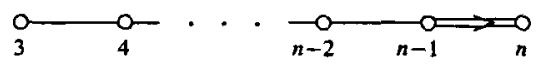

$D_{k} \times B_{n-k}$

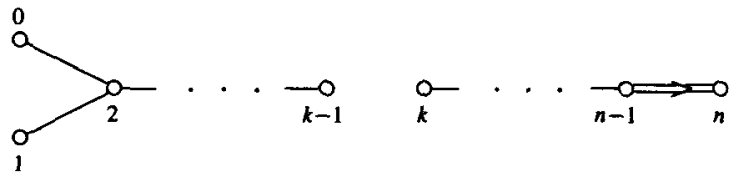

$D_{n-1} \times A_{1}$

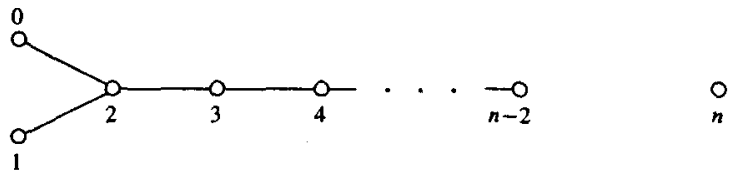

$D_{n}$

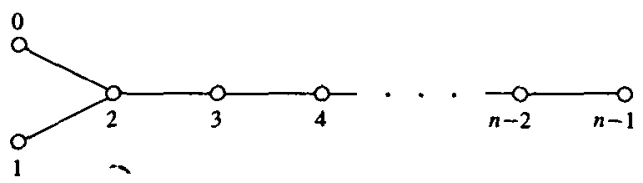

$B_{n-1}$

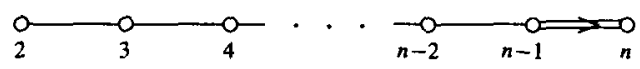

FIGURE 2. Maximal subdiagrams for $B_{n}$.

Because the highest root is $e_{1}+e_{2}$, the two roots making up $A_{1} \times A_{1}$ (in the base we have chosen) are $\left\{e_{1} \pm e_{2}\right\}$, which for simplicity are referred to as $D_{2}$. If any of vertices 3 through $n-2$ are removed, say vertex $k$, we have type $D_{k} \times B_{n-k}$, where $k \geq 3, n-k \geq 2$ and $D_{3}$ is understood to be the obvious root system. It has base

$$
\left\{\alpha_{0}, \alpha_{1}, \ldots, \alpha_{k-1}\right\} \cup\left\{\alpha_{k+1}, \ldots, \alpha_{n}\right\}
$$

which in terms of $\Delta$ may be expressed as

$$
\left\{e_{1} \pm e_{2}, e_{i}-e_{i+1}: 2 \leq i \leq k-1\right\} \cup\left\{e_{i}-e_{i+1}, e_{n}: k+1 \leq i \leq n-1\right\} .
$$

The Weyl group acts as the group of permutations and sign changes of the set $\left\{e_{1}, \ldots, e_{n}\right\}$. Thus any set of positive roots associated with the subroot systems 
of type $D_{k} \times B_{n-k}$ is of the form

$$
\left\{e_{i} \pm e_{j}: i<j ; i, j \in J_{1}\right\} \cup\left\{e_{l}, e_{i} \pm e_{j}: i<j ; i, j, l \in J_{2}\right\},
$$

where $J_{1}$ and $J_{2}$ are disjoint subsets of $\{1, \ldots, n\}$ of sizes $k$ and $n-k$ respectively.

If vertex $n-1$ is removed the subroot system is type $A_{1} \times D_{n-1}$ and the root in $A_{1}$ is short. The sets of positive roots associated with this type of maximal subroot system are of the form

$$
\left\{e_{i}\right\} \cup\left\{e_{l} \pm e_{j}: l<j ; l, j \neq i\right\} .
$$

When vertex $n$ is removed we are left with type $D_{n}$ and positive roots

$$
\left\{e_{i} \pm e_{j}: 1 \leq i<j \leq n\right\}
$$

If vertices 0 and 1 are both removed we are left with the maximal system $B_{n-1}$ and the sets of positive roots are Weyl conjugates of

$$
\left\{e_{l}, e_{i} \pm e_{j}: 1<i<j \leq n, l \neq 1\right\}
$$

and thus are of the form

$$
\left\{e_{l}, e_{i} \pm e_{j}: i<j ; i, j, l \neq n_{0}\right\} .
$$

If any other two (or more) vertices are removed from the extended graph we clearly do not have a maximal subroot system.

Notice that of all these maximal subroot systems only types $D_{k} \times B_{n-k}$ and $A_{1} \times D_{n-1}$ are also of maximal rank.

2.3. Summary of maximal subroot systems In the charts which follow $J_{1}$ and $J_{2}$ denote disjoint subsets of $\{1, \ldots, n\}$ in types $B_{n}, C_{n}$ and $D_{n}$; and disjoint subsets of $\{1, \ldots, n+1\}$ in type $A_{n}$.

$\begin{array}{lll}\text { Type } & \begin{array}{l}\text { Maximal } \\ \text { subroot } \\ \text { systems }\end{array} & \text { Positive roots of the maximal subroot systems } \\ \mathbf{A}_{n} & A_{n-1} & \left\{e_{i}-e_{j}: 1 \leq i<j \leq n+1 ; i, j \neq n_{0}\right\} \\ & A_{k} \times A_{n-k-1} & \left\{e_{i}-e_{j}: i<j ; i, j \in J_{1}\right\} \cup\left\{e_{i}-e_{j}: i<j ; i, j \in J_{2}\right\}, \\ & \text { where }\left|J_{1}\right|=k+1 \geq 2,\left|J_{2}\right|=n-k \geq 2 \\ \mathbf{B}_{n} & B_{n-1} & \left\{e_{l}, e_{i} \pm e_{j}: i<j ; i, j, l \neq n_{0}\right\} \\ & D_{n} & \left\{e_{i} \pm e_{j}: 1 \leq i<j \leq n\right\} \\ & D_{k} \times B_{n-k} & \left\{e_{i} \pm e_{j}: i<j ; i, j \in J_{1}\right\} \cup\left\{e_{l}, e_{i} \pm e_{j}: i<j ; i, j, l \in J_{2}\right\}, \\ & \text { where }\left|J_{1}\right|=k \geq 2,\left|J_{2}\right|=n-k \geq 2 \\ & A_{1} \times D_{n-1} & \left\{e_{i}\right\} \cup\left\{e_{l} \pm e_{j}: l<j ; l, j \neq i\right\}\end{array}$




$$
\begin{array}{lll}
\mathbf{C}_{n} & A_{n-1} & \left\{s_{i} e_{i}-s_{j} e_{j}: 1 \leq i<j \leq n\right\}, \text { where } s_{j}= \pm 1 \\
& C_{k} \times C_{n-k} & \left\{2 e_{l}, e_{i} \pm e_{j}: i<j ; i, j, l \in J_{1}\right\} \\
& & \cup\left\{2 e_{l}, e_{i} \pm e_{j}: i<j ; i, j, l \in J_{2}\right\}, \\
& & \text { where }\left|J_{1}\right|=k \geq 1,\left|J_{2}\right|=n-k \geq 1 \\
& & \left\{e_{i} \pm e_{j}: i<j ; i, j \neq n_{0}\right\} \\
\mathbf{D}_{n} & D_{n-1} & \left\{s_{i} e_{i}-s_{j} e_{j}: 1 \leq i<j \leq n\right\}, \text { where } s_{j}= \pm 1 \\
& A_{n-1} & \text { and an even number of } s_{j}=-1 \\
& & \\
D_{k} \times D_{n-k} & \left\{e_{i} \pm e_{j}: i<j ; i, j \in J_{1}\right\} \cup\left\{e_{i} \pm e_{j}: i<j ; i, j \in J_{2}\right\}, \\
& & \text { where }\left|J_{1}\right|=k \geq 2,\left|J_{2}\right|=n-k \geq 2
\end{array}
$$

Here $D_{2}$ is understood to mean $\left\{e_{1} \pm e_{2}\right\}$ and $C_{1}=\left\{2 e_{i}\right\} . C_{2}$ and $D_{3}$ are the obvious root systems.

\section{Upper bounds for the trace function}

In this section we establish the sufficiency of the choice of $s$ in our main result. Each Lie group type must be handled separately, taking into account the possible choices for $\Phi^{+}(g)$.

THEOREM 3.1. Let $G$ be a compact, connected, simple Lie group of type $A_{n}, B_{n}$, $C_{n}$ or $D_{n}$. For every $g \notin Z(G)$ there is a constant $c(g)$ such that

$$
\left|\frac{\operatorname{Tr} \lambda(g)}{\operatorname{deg} \lambda}\right| \leq c(g)(\operatorname{deg} \lambda)^{-s}
$$

for all $\lambda \in \widehat{G}$ provided

$$
s \leq \begin{cases}1 /(n-1) & \text { if } G \text { is type } A_{n-1} \text { or } D_{n} \\ 1 /(2 n-1) & \text { if } G \text { is type } B_{n} \\ 2 /(2 n-1) & \text { if } G \text { is type } C_{n}, n \neq 3 \\ 1 / 3 & \text { if } G \text { is type } C_{3} .\end{cases}
$$

PROOF. Inequality (2.3) together with the Weyl dimension formula (2.1) show that it is sufficient to prove that there is some constant $c$ such that for all $w \in W$ and representations $\lambda$,

$$
\frac{\left|\prod_{\alpha \in \Phi^{+}(g)}(\rho+\lambda, w(\alpha))\right|}{\prod_{\alpha \in \Phi^{+}}(\rho+\lambda, \alpha)^{1-s}} \leq c .
$$


Indeed, as $\alpha \in \Phi(g)$ if and only if $w(\alpha) \in \Phi\left(w^{-1}(g)\right)$, it suffices to prove there is a constant $c$ such that

$$
\frac{\prod_{\alpha \in \Phi^{++}}(\rho+\lambda, \alpha)}{\prod_{\alpha \in \Phi^{+}}(\rho+\lambda, \alpha)^{1-s}}=\prod_{\alpha \in \Phi^{+\prime}}(\rho+\lambda, \alpha)^{s} \prod_{\alpha \in \Phi^{+} \backslash \Phi^{++}}(\rho+\lambda, \alpha)^{s-1} \leq c
$$

whenever $\Phi^{+\prime}$ is the set of positive roots of some maximal subroot system, and this is what we show in each case.

Throughout this proof we assume $\rho+\lambda$ can be expressed in terms of the fundamental dominant weights as $\sum_{i=1}^{n} m_{i} \lambda_{i}$. We also assume $m_{k}=\max _{i=1 \ldots, n} m_{i}$. The letter $c$ denotes a constant which may vary from one line to another.

One common technique we use is an induction argument. We often partition $\Phi^{+}$ (and $\Phi^{+\prime}$ ) into two sets, one of which is a positive root system (subroot system) of smaller type. The product we need to study corresponding to these roots of smaller type are handled by the induction assumption. Another common technique is to count the number of positive roots $\alpha$, from some appropriate set, such that $(\rho+\lambda, \alpha)$ is (essentially) maximal and see that there are enough of these terms occuring in the product with a negative exponent to make the product suitably small. Both these ideas are used in Case 1.1 below (when the maximal subroot system is type $A_{n-1}$ in type $A_{n}$ ). In other cases, the arguments are slightly more delicate, but always they are of an elementary, combinatorial nature.

Type $A_{n}$

Case 1.1 Maximal subroot system is type $A_{n-1}$.

We proceed by induction on $n$. If $n=1$, then $\Phi^{+\prime}$ is empty and consequently $s=1$ suffices. So assume inductively that (3.1) is satisfied with $s=1 /(n-1)$ whenever $\Phi^{+}$is the set of positive roots of type $A_{n-1}$ and $\Phi^{+\prime}$ is the set of positive roots of a subroot system of type $A_{n-2}$.

Let $\Phi^{+}$be the set of positive roots of type $A_{n}$ and let $\Phi^{+\prime}$ be the set of positive roots of a subroot system of type $A_{n-1} ; \Phi^{+\prime}$ is a set of the form $\left\{e_{i}-e_{j}: 1 \leq i<j \leq\right.$ $\left.n+1 ; i, j \neq n_{0}\right\}$.

First assume $k \leq n_{0}-1$ (which implies, in particular, that $n_{0} \neq 1$ ). Partition $\Phi^{+}$ as $\Phi_{1}^{+} \cup \Phi_{2}^{+}$with

$$
\Phi_{1}^{+}=\left\{e_{i}-e_{j}: 2 \leq i<j \leq n+1\right\} \text { and } \Phi_{2}^{+}=\left\{e_{1}-e_{j}: 2 \leq j \leq n+1\right\} .
$$

Similarly partition $\Phi^{+\prime}$ as $\Phi_{1}^{+\prime} \cup \Phi_{2}^{+\prime}$, where

$$
\Phi_{1}^{+\prime}=\left\{e_{i}-e_{j}: 2 \leq i<j \leq n+1 ; i, j \neq n_{0}\right\}
$$

and

$$
\Phi_{2}^{+\prime}=\left\{e_{1}-e_{j}: 2 \leq j \leq n+1 ; j \neq n_{0}\right\}
$$


The set $\Phi_{1}^{+}$may be viewed as the positive roots of type $A_{n-1}$ and $\Phi_{1}^{+\prime}$ as the positive roots of a subroot system of type $A_{n-2}$ (considering the vectors to be in $\mathbb{R}^{n}$ by omitting the first (zero) coordinate). When $\alpha \in \Phi_{1}^{+}$, then $(\rho+\lambda, \alpha)$ is equal to $\left(\sum_{i=2}^{n} m_{i} \lambda_{i}, \alpha\right)$, thus the inductive hypothesis may be applied to conclude that if $s \leq 1 /(n-1)$, then

$$
\prod_{\alpha \in \Phi_{1}^{++}}(\rho+\lambda, \alpha)^{s} \prod_{\alpha \in \Phi_{1}^{+} \backslash \Phi_{1}^{+\prime}}(\rho+\lambda, \alpha)^{s-1} \leq c .
$$

Since the cardinality of $\Phi_{2}^{+\prime}$ is $n-1$ we clearly have

$$
\prod_{\alpha \in \Phi_{2}^{+\prime}}(\rho+\lambda, \alpha)^{s} \leq c m_{k}^{s(n-1)} .
$$

Recall that $e_{1}-e_{n_{0}}=\lambda_{1}+\cdots+\lambda_{n_{0}-1}$. As $k \leq n_{0}-1$ this means that $\left(\rho+\lambda, e_{1}-e_{n_{0}}\right) \geq$ $m_{k}$, and since $e_{1}-e_{n_{0}} \in \Phi_{2}^{+} \backslash \Phi_{2}^{+\prime}$ we obtain the inequality

$$
\prod_{\alpha \in \Phi_{2}^{+} \backslash \Phi_{2}^{++}}(\rho+\lambda, \alpha)^{s-1} \leq m_{k}^{s-1}
$$

Therefore,

$$
\prod_{\alpha \in \Phi_{2}^{+^{+}}}(\rho+\lambda, \alpha)^{s} \prod_{\alpha \in \Phi_{2}^{+} \backslash \Phi_{2}^{++}}(\rho+\lambda, \alpha)^{s-1} \leq c m_{k}^{s(n-1)+s-1}
$$

This is bounded if $s(n-1)+s-1 \leq 0$, that is, when $s \leq 1 / n$, giving the desired result.

Otherwise $k \geq n_{0}$ (and $n_{0} \neq n+1$ ). In this case we partition $\Phi^{+\prime}$ into $\Phi_{1}^{+\prime} \cup \Phi_{2}^{+\prime}$, where $\Phi_{1}^{+\prime}$ is the subset of $\Phi^{+\prime}$ consisting of all the words $e_{i}-e_{j}$ with $i, j \neq n+1$, and

$$
\Phi_{2}^{+\prime}=\left\{e_{j}-e_{n+1}: 1 \leq j \leq n ; j \neq n_{0}\right\}
$$

Similarly partition $\Phi^{+}$so that $\Phi_{2}^{+} \backslash \Phi_{2}^{+\prime}=\left\{e_{n_{0}}-e_{n+1}\right\}$. Again the inductive hypothesis can be applied to the factors of the product corresponding to $\alpha \in \Phi_{1}^{+}$and $\Phi_{1}^{+1}$, and this observation reduces the problem to proving

$$
\prod_{\alpha \in \Phi_{2}^{++}}(\rho+\lambda, \alpha)^{s} \prod_{\alpha \in \Phi_{2}^{+} \backslash \Phi_{2}^{++}}(\rho+\lambda, \alpha)^{s-1} \leq c
$$

for $s \leq 1 / n$. As

$$
\left(\rho+\lambda, e_{n_{0}}-e_{n+1}\right)=\left(\rho+\lambda, \lambda_{n_{0}}+\cdots+\lambda_{n}\right) \geq m_{k}
$$

the required inequality can be established in the same manner as the first part. 
Case 1.2 Maximal subroot system is type $A_{k} \times A_{n-k-1}$, where $k, n-k-1 \geq 1$.

We again proceed by induction on $n$. Notice that a maximal subroot system of this type is not found in type $A_{1}$ or $A_{2}$, and consequently the initial step of the inductive hypothesis is with $n=3$ and $\Phi^{+\prime}$ the set of positive roots of type $A_{1} \times A_{1}$. We leave it to the reader to verify the hypothesis for this initial condition.

We assume inductively that (3.1) holds with $s=1 /(n-1)$ whenever $\Phi^{+}$is the set of positive roots of type $A_{n-1}$ and $\Phi^{+\prime}$ is type $A_{k} \times A_{n-k-2}$ for some $k$ and $n-k-2 \geq 1$, and proceed to verify the induction step for type $A_{n}$.

From Section 2.3 we know that any set of positive roots of type $A_{k} \times A_{n-k-1}$ in $A_{n}$ is of the form $\Phi^{+\prime}=\Phi_{1}^{+\prime} \cup \Phi_{2}^{+\prime}$, where

$$
\begin{aligned}
& \Phi_{1}^{+\prime}=\left\{e_{i}-e_{j}: i<j ; i, j \in J_{1}\right\}, \\
& \Phi_{2}^{+\prime}=\left\{e_{i}-e_{j}: i<j ; i, j \in J_{2}\right\}
\end{aligned}
$$

and $J_{1}, J_{2}$ are disjoint sets whose union is $\{1, \ldots, n+1\}$, of sizes $k+1$ and $n-k$ respectively. Without loss of generality we may assume $1 \in J_{1}$.

Let

$$
\Psi_{1}^{\prime}=\left\{e_{i}-e_{j}: 1<i<j ; i, j \in J_{1}\right\}
$$

( $\Psi_{1}^{\prime}$ is taken to be empty if the cardinality of $J_{1}$ is two) and $\Psi_{2}^{\prime}=\Phi_{1}^{+\prime} \backslash \Psi_{1}^{\prime}$. Let $\Psi_{1}$ be the set of words $e_{i}-e_{j}, i<j$, on the letters $\{2, \ldots, n+1\}$, and $\Psi_{2}=\left\{e_{1}-e_{j}: j \neq 1\right\}$. Then $\Psi_{1}$ may be viewed as the set of positive roots of type $A_{n-1}$, with $\Psi_{1}^{\prime} \cup \Phi_{2}^{+\prime}$ a subroot system of type $A_{k-1} \times A_{n-k-1}$. Thus the inductive hypothesis may be applied to yield

$$
\prod_{\alpha \in \Psi_{1}^{\prime} \cup \Phi_{2}^{+\prime}}(\rho+\lambda, \alpha)^{s} \prod_{\alpha \in \Psi_{1} \backslash \Psi_{1}^{\prime} \cup \Phi_{2}^{+\prime}}(\rho+\lambda, \alpha)^{s-1} \leq c
$$

when $s \leq 1 /(n-1)$. (If $\Psi_{1}^{\prime}$ is empty, then this is actually Case 1.1 which has already been done.)

It remains to prove that for $\Psi_{2}^{\prime}=\left\{e_{1}-e_{j}: j \in J_{1} \backslash\{1\}\right\}$ and $s \leq 1 / n$,

$$
\prod_{\alpha \in \Psi_{2}^{\prime}}(\rho+\lambda, \alpha)^{s} \prod_{\alpha \in \Psi_{2} \backslash \Psi_{2}^{\prime}}(\rho+\lambda, \alpha)^{s-1} \leq c .
$$

If there exists some $j \in J_{2}$ such that $j \geq k+1$, then for some $\alpha \in \Psi_{2} \backslash \Psi_{2}^{\prime}$

$$
(\rho+\lambda, \alpha)=\left(\rho+\lambda, e_{1}-e_{j}\right)=\left(\rho+\lambda, \lambda_{1}+\cdots+\lambda_{j-1}\right) \geq m_{k} .
$$

Combining this with the fact that the cardinality of $J_{1}$ is at most $n-1$ we obtain the inequalities

$$
\prod_{\alpha \in \Psi_{2}^{\prime}}(\rho+\lambda, \alpha)^{s} \prod_{\alpha \in \Psi_{2} \backslash \Psi_{2}^{\prime}}(\rho+\lambda, \alpha)^{s-1} \leq c m_{k}^{s\left(\left|S_{1}\right|-1\right)} m_{k}^{s-1} \leq c
$$


when $s \leq 1 / n$.

Otherwise, $n+1 \in J_{1}$ and there is some $j \leq k$ which belongs to $J_{2}$. (Indeed, all $j \in J_{2}$ must satisfy $j \leq k$.) Redefine

$$
\begin{aligned}
& \Psi_{1}^{\prime}=\left\{e_{i}-e_{j}: 1 \leq i<j \leq n ; i, j \in J_{1}\right\}, \\
& \Psi_{1}=\left\{e_{i}-e_{j}: 1 \leq i<j \leq n\right\}
\end{aligned}
$$

and $\Psi_{2}, \Psi_{2}^{\prime}$ correspondingly. The argument now follows from the fact that $(\rho+\lambda, \alpha) \geq$ $m_{k}$ for $\alpha=e_{j}-e_{n+1} \in \Psi_{2} \backslash \Psi_{2}^{\prime}$.

Type $B_{n}$

Case 2.1 Maximal subroot system is type $B_{n-1}$.

The maximal subroot system $\Phi^{+\prime}=\left\{e_{l}, e_{i} \pm e_{j}: i<j ; i, j, l \neq n_{0}\right\}$. We consider the cases $n_{0}=1$ and $n_{0} \neq 1$ separately and assume $s \leq 1 /(2 n-1)$.

$n_{0}=1:$ Notice $(\rho+\lambda, \alpha)=O\left(m_{k}\right)$ for all $\alpha=e_{1}, e_{1}+e_{j}$ and these roots all belong to $\Phi^{+} \backslash \Phi^{+\prime}$. Also, $\left|\Phi^{+\prime}\right|=(n-1)^{2}$, and thus

$$
\prod_{\alpha \in \Phi^{++}}(\rho+\lambda, \alpha)^{s} \prod_{\alpha \in \Phi^{+} \backslash \Phi^{+}}(\rho+\lambda, \alpha)^{s-1} \leq m_{k}^{s(n-1)^{2}} m_{k}^{(s-1) n} .
$$

Since

$$
\frac{1}{2 n-1} \leq \frac{n}{n^{2}-n+1}
$$

it follows that (3.2) is bounded whenever $s \leq 1 /(2 n-1)$.

$n_{0} \neq 1$ : Here we proceed by induction, leaving the initial step with $n=3$ to the reader. The words from $\Phi^{+\prime}$ in $\Phi^{+}$with letters from $\{2, \ldots, n\}$ are the positive roots of a subroot system of type $B_{n-2}$ in type $B_{n-1}$. Thus the inductive hypothesis reduces the problem to consideration of

$$
\prod_{\alpha \in \Psi^{\prime}}(\rho+\lambda, \alpha)^{s} \prod_{\alpha \in \Psi \backslash \Psi^{\prime}}(\rho+\lambda, \alpha)^{s-1},
$$

where $\Psi^{\prime}$ and $\Psi$ are the remaining roots in $\Phi^{+\prime}$ and $\Phi^{+}$respectively.

Set $a_{j}=\max \left\{m_{l}: l<j\right\}$. Notice that $\left\{a_{j}\right\}$ is an increasing sequence and that $\left(\rho+\lambda, e_{1}-e_{j}\right)=O\left(a_{j}\right)$. Also, both $\left(\rho+\lambda, e_{1}\right)$ and $\left(\rho+\lambda, e_{1}+e_{j}\right)$ are $O\left(m_{k}\right)$. As $\Psi^{\prime}=\left\{e_{1}, e_{1} \pm e_{j}: j \neq 1, n_{0}\right\}$, this implies

$$
\begin{aligned}
\prod_{\alpha \in \Psi^{\prime}}(\rho+\lambda, \alpha)^{s} & =\left(\rho+\lambda, e_{1}\right)^{s} \prod_{\alpha=e_{1}+e_{j}, j \neq 1, n_{0}}(\rho+\lambda, \alpha)^{s} \prod_{\alpha=e_{1}-e_{j}, j \neq 1, n_{0}}(\rho+\lambda, \alpha)^{s} \\
& \leq c m_{k}^{s(n-1)} \prod_{j=2}^{n_{0}-1} a_{j}^{s} \prod_{j=n_{0}+1}^{n} a_{j}^{s} \leq c m_{k}^{s(n-1)} a_{n_{0}}^{s\left(n_{0}-2\right)} m_{k}^{s\left(n-n_{0}\right)} \\
& \leq c m_{k}^{s\left(2 n-n_{0}-1\right)} a_{n_{0}}^{s\left(n_{0}-2\right)} .
\end{aligned}
$$


Moreover, $\Psi \backslash \Psi^{\prime}=\left\{e_{1} \pm e_{n_{0}}\right\}$, therefore

$$
\prod_{\alpha \in \Psi \backslash \Psi^{\prime}}(\rho+\lambda, \alpha)^{s-1}=c m_{k}^{s-1} a_{n_{0}}^{s-1} .
$$

Hence

$$
\prod_{\alpha \in \Psi^{\prime}}(\rho+\lambda, \alpha)^{s} \prod_{\alpha \in \Psi^{\prime} \Psi^{\prime}}(\rho+\lambda, \alpha)^{s-1} \leq c m_{k}^{s\left(2 n-n_{0}\right)-1} a_{n_{0}}^{s\left(n_{0}-1\right)-1},
$$

and noting that both exponents are negative completes the argument.

Case 2.2 Maximal subroot system is type $D_{n}$.

In this case $\Phi^{+\prime}=\left\{e_{i} \pm e_{j}: 1 \leq i<j \leq n\right\}$ and therefore $\Phi^{+} \backslash \Phi^{+\prime}$ is the set of all words of length one in $\Phi^{+}$. Let $b_{i}=\max \left\{m_{l}: l \geq i\right\}$. Then $(\rho+\lambda, \alpha)=O\left(b_{i}\right)$ if $\alpha=e_{i}$ or $e_{i}+e_{j}$ for any $j>i$. Also, $\left(\rho+\lambda, e_{i}-e_{j}\right) \leq O\left(b_{i}\right)$ whenever $j>i$. Thus

$$
\begin{aligned}
\prod_{\alpha \in \Phi^{+}}(\rho+\lambda, \alpha)^{s} \prod_{\alpha \in \Phi^{+} \backslash \Phi^{++}}(\rho+\lambda, \alpha)^{s-1} & =\prod_{\alpha=e_{i} \pm e_{j}, i<j}(\rho+\lambda, \alpha)^{s} \prod_{i=1}^{n}\left(\rho+\lambda, e_{i}\right)^{s-1} \\
& \leq c \prod_{i=1}^{n-1} b_{i}^{2 s(n-i)} \prod_{i=1}^{n} b_{i}^{s-1}=c \prod_{i=1}^{n} b_{i}^{s(2 n-2 i+1)-1},
\end{aligned}
$$

and this is clearly bounded for $s \leq 1 /(2 n-1)$.

Case 2.3 Maximal subroot system is type $A_{1} \times D_{n-1}$.

The argument is essentially the same as Case 2.2 .

Case 2.4 Maximal subroot system is type $D_{m} \times B_{n-m} ; m, n-m \geq 2$.

The positive roots of type $D_{m}$ in type $B_{m}$ were already treated in Case 2.2, so it suffices to show

$$
P \equiv\left|\prod_{\alpha \in \Psi^{\prime}}(\rho+\lambda, \alpha)^{s} \prod_{\alpha \in \Psi}(\rho+\lambda, \alpha)^{s-1}\right|
$$

is bounded when

$$
\Psi^{\prime}=\left\{e_{l}, e_{i} \pm e_{j}: i<j ; i, j, l \in J_{2}\right\} \quad \text { and } \quad \Psi=\left\{e_{i} \pm e_{j}: i \in J_{1}, j \in J_{2}\right\} .
$$

We consider the cases $1 \in J_{1}$ and $1 \in J_{2}$ separately. The argument is much easier when $1 \in J_{1}$ and hinges on the fact that in this case $\Psi \supseteq\left\{e_{1} \pm e_{j}: j \in J_{2}\right\}$. Thus

$$
P \leq \prod_{i \in J_{2}}\left(\rho+\lambda, e_{i}\right)^{s} \prod_{i<j \in J_{2}}\left(\rho+\lambda, e_{i} \pm e_{j}\right)^{s} \prod_{j \in J_{2}}\left(\rho+\lambda, e_{1} \pm e_{j}\right)^{s-1} .
$$

Let $b_{i}=\max \left\{m_{l}: l \geq i\right\}$ and $a_{i}=\max \left\{m_{l}: l<i\right\}$. With this notation, for $i<j$ we have

$$
\left(\rho+\lambda, e_{i}+e_{j}\right)=O\left(b_{i}\right), \quad\left(\rho+\lambda, e_{i}\right)=O\left(b_{i}\right)
$$




$$
\left(\rho+\lambda, e_{i}-e_{j}\right) \leq O\left(a_{j}\right), \quad\left(\rho+\lambda, e_{1}-e_{j}\right)=O\left(a_{j}\right)
$$

Hence we can further bound $P$ by

$$
P \leq c \prod_{i \in J_{2}} b_{i}^{s} b_{i}^{s\left(\left|J_{2}\right|-1\right)} \prod_{j \in J_{2}} a_{j}^{s\left(\left|J_{2}\right|-1\right)} b_{1}^{(s-1)\left|J_{2}\right|} \prod_{j \in J_{2}} a_{j}^{s-1} \leq c b_{1}^{s\left(\left|J_{2}\right|^{2}+\left|J_{2}\right|\right)-\left|J_{2}\right|} \prod_{j \in J_{2}} a_{j}^{s\left|J_{2}\right|-1} .
$$

The final product is bounded over all $\lambda$ since $\left|J_{2}\right| \leq n-2$ and $s \leq 1 /(2 n-1)$.

Now assume $1 \in J_{2}$. Here a further induction argument is useful. Partition $\Psi^{\prime}$ as $X_{1} \cup X_{2}$ and $\Psi$ as $Y_{1} \cup Y_{2}$, where

$$
X_{1}=\left\{e_{l}, e_{i} \pm e_{j}: i<j, 1 \neq i, j, l \in J_{2}\right\}, \quad X_{2}=\left\{e_{1}, e_{1} \pm e_{j}: 1 \neq j \in J_{2}\right\}
$$

and

$$
Y_{1}=\left\{e_{i} \pm e_{j}: i \in J_{1}, 1 \neq j \in J_{2}\right\}, \quad Y_{2}=\left\{e_{1} \pm e_{i}: i \in J_{1}\right\}
$$

and assume inductively that

$$
\left|\prod_{\alpha \in X_{1}}(\rho+\lambda, \alpha)^{s} \prod_{\alpha \in Y_{1}}(\rho+\lambda, \alpha)^{s-1}\right| \leq c
$$

for $s \leq 1 /(2 n-1)$. (The initial case is left for the reader). We need to check that

$$
\left|\prod_{\alpha \in X_{2}}(\rho+\lambda, \alpha)^{s} \prod_{\alpha \in Y_{2}}(\rho+\lambda, \alpha)^{s-1}\right| \leq c
$$

to complete the induction step. Since $\left(\rho+\lambda, e_{1}+e_{i}\right)=O\left(m_{k}\right)$ for all $i \in J_{1}$, and $\left(\rho+\lambda, e_{1}-e_{i}\right)^{s-1} \leq 1$, the product above is bounded by $m_{k}^{s\left|X_{2}\right|} m_{k}^{(s-1)\left|J_{1}\right|}$. As $\left|X_{2}\right|=2\left|J_{2}\right|-1$ and $J_{1}$ has at least two elements the desired result is obtained.

This completes type $B_{n}$.

\section{Type $C_{n}$}

Case 3.1 Maximal subroot system is type $A_{n-1}$.

When $k=n$ then $\left(\rho+\lambda, 2 e_{i}\right)=O\left(m_{k}\right)$ for all $i=1, \ldots, n$ and as these roots belong to $\Phi^{+} \backslash \Phi^{+\prime}$ it follows that for $s \leq 2 /(2 n-1)$,

$$
P=\prod_{\alpha \in \Phi^{++}}(\rho+\lambda, \alpha)^{s} \prod_{\alpha \in \Phi^{+} \backslash \Phi^{++}}(\rho+\lambda, \alpha)^{s-1} \leq m_{k}^{s\left(\begin{array}{c}
n \\
2
\end{array}\right)} m_{k}^{(s-1) n} \leq c .
$$

When $k \neq n$ we proceed inductively. The words from $\Phi^{+}$and $\Phi^{+\prime}$ built on the letters $\{2, \ldots, n\}$ form a subroot system of type $A_{n-2}$ in $C_{n-1}$ and thus our standard induction argument reduces the problem to showing that

$$
P=\prod_{\alpha \in \Psi^{\prime}}(\rho+\lambda, \alpha)^{s} \prod_{\alpha \in \Psi \backslash \Psi^{\prime}}(\rho+\lambda, \alpha)^{s-1} \leq c,
$$


where $\Psi^{\prime}$ and $\Psi$ are the remaining words of $\Phi^{+\prime}$ and $\Phi^{+}$respectively.

As $\Psi^{\prime}$ contains only one of $e_{1} \pm e_{n}$, it follows that $(\rho+\lambda, \alpha)=O\left(m_{k}\right)$ for at least two $\alpha \in \Psi \backslash \Psi^{\prime}$, namely, $\alpha=2 e_{1}$ and the one of $e_{1} \pm e_{n}$ which is not in $\Psi^{\prime}$. Furthermore, $\left|\Psi^{\prime}\right|=n-1$, hence

$$
P \leq m_{k}^{s(n-1)} m_{k}^{2(s-1)},
$$

and this is certainly bounded for $s \leq 2 /(2 n-1)$.

Case 3.2 Maximal subroot system is type $C_{1} \times C_{n-1}$.

This case is much more delicate than any of the others. When $n=3$ it can be done by explicit calculation and we leave this for the reader. So we begin with $n \geq 4$ and take $s \geq 2 /(2 n-1)$.

As the maximal subroot system is

$$
\Phi^{+\prime}=\left\{2 e_{i_{0}}\right\} \cup\left\{2 e_{l}, e_{i} \pm e_{j}: i<j ; i, j, l \neq i_{0}\right\},
$$

(3.1) can be written as

$$
P=\prod_{l=1}^{n}\left(\rho+\lambda, 2 e_{l}\right)^{s} \prod_{i<j \neq i_{0}}\left(\rho+\lambda, e_{i} \pm e_{j}\right)^{s} \prod_{j \neq i_{0}}\left|\left(\rho+\lambda, e_{i_{0}} \pm e_{j}\right)\right|^{s-1} .
$$

Let $b_{i}=\max \left\{m_{l}: l \geq i\right\}$. When $i<j$ then $\left(\rho+\lambda, e_{i}+e_{j}\right)=O\left(b_{i}\right)=\left(\rho+\lambda, 2 e_{i}\right)$. Thus

$$
P=c \prod_{i=1}^{n} b_{i}^{s} \prod_{i<i_{0}} b_{i}^{s(n-i-1)} \prod_{i>i_{0}} b_{i}^{s(n-i)} \prod_{j<i_{0}} b_{j}^{s-1} b_{i_{0}}^{\left(n-i_{0}\right)(s-1)} Q
$$

where

$$
Q=\prod_{i<j \neq i_{0}}\left(\rho+\lambda, e_{i}-e_{j}\right)^{s} \prod_{j \neq i_{0}}\left|\left(\rho+\lambda, e_{i_{0}}-e_{j}\right)\right|^{s-1} .
$$

Notice that $Q$ is the product we considered for the problem of the maximal subroot system of type $A_{n-2}$ in type $A_{n-1}$ (Case 1.1), and thus is bounded provided $s \leq$ $1 /(n-1)$. This is true in our situation since we have the stronger inequality $s \leq$ $2 /(2 n-1)$.

Simplifying, and using the fact that when $i>i_{0}$ then $b_{i} \leq b_{i_{0}}$, we obtain

$$
P \leq c b_{i_{0}}^{s+\left(n-i_{0}\right)(s-1)} \prod_{i<i_{0}} b_{i}^{s(n-i+1)-1} \prod_{i>i_{0}} b_{i_{0}}^{s(n-i+1)} Q
$$

and hence

$$
P \leq c b_{i_{0}}^{s\left(n-i_{0}+1+\left(n-i_{0}+1\right)\left(n-i_{0}\right) / 2\right)-\left(n-i_{0}\right)} \prod_{i<i_{0}} b_{i}^{s(n-i+1)-1} Q .
$$


To continue, we split the problem into two cases. First, suppose $k \geq i_{0}$. Then $b_{i}=m_{k}$ whenever $i \leq i_{0}$. Recall also that $Q$ is bounded, thus

$$
P \leq c m_{k}^{s\left(n-i_{0}+1+\left(n-i_{0}+1\right)\left(n-i_{0}\right) / 2\right)-\left(n-i_{0}\right)} \prod_{i<i_{0}} m_{k}^{s(n-i+1)-1} .
$$

Routine calculations reduce this to the inequality

$$
P \leq m_{k}^{s\left(n^{2}+n\right) / 2-n+1}
$$

which one can check is bounded for our choices of $n$ and $s$.

Now, suppose $k<i_{0}$. A standard argument with inequalities shows that the exponent of $b_{i_{0}}$ in (3.4) is negative (for $s \leq 2 /(2 n-1)$ ) if $i_{0} \neq n$. Also, $s(n-i+1)-1 \leq 0$ if $i>1$. Consequently,

$$
P \leq \begin{cases}c b_{n}^{s} b_{1}^{s n-1} b_{n-1}^{2 s-1} Q & \text { if } i_{0}=n \\ c b_{1}^{s n-1} Q & \text { if } i_{0} \neq n .\end{cases}
$$

We factor $Q$ as

$$
Q=\prod_{j \neq 1, i_{0}}\left(\rho+\lambda, e_{1}-e_{j}\right)^{s}\left(\rho+\lambda, e_{1}-e_{i_{0}}\right)^{s-1} Q_{1},
$$

where

$$
Q_{1}=\prod_{i<j \neq 1, i_{0}}\left(\rho+\lambda, e_{i}-e_{j}\right)^{s} \prod_{j \neq 1, i_{0}}\left|\left(\rho+\lambda, e_{j}-e_{i_{0}}\right)\right|^{s-1} .
$$

$Q_{1}$ is bounded being the product we consider for the problem of a maximal subroot system of type $A_{n-3}$ in type $A_{n-2}$ (on the letters $\{2, \ldots, n\}$; note that the assumption $k<i_{0}$ implies $\left.i_{0} \neq 1\right)$. Also, as $k<i_{0},\left(\rho+\lambda, e_{1}-e_{i_{0}}\right)=O\left(m_{k}\right)$, thus

$$
P \leq \begin{cases}c b_{n}^{s} b_{1}^{s n-1} b_{n-1}^{2 s-1} m_{k}^{s(n-2)} m_{k}^{s-1} Q_{1} & \text { if } i_{0}=n \\ c b_{1}^{s n-1} m_{k}^{s(n-2)} m_{k}^{s-1} Q_{1} & \text { if } i_{0} \neq n .\end{cases}
$$

But $b_{n} \leq b_{n-1}$ and $\mathrm{b}_{1}=m_{k}$, hence

$$
P \leq \begin{cases}c b_{n-1}^{3 s-1} m_{k}^{s(2 n-1)-2} & \text { if } i_{0}=n \\ c m_{k}^{s(2 n-1)-2} & \text { if } i_{0} \neq n .\end{cases}
$$

As $n \geq 4$ we have $s \leq 1 / 3$, and thus $P$ is bounded in either case.

Case 3.3 Maximal subroot system is type $C_{k} \times C_{n-k} ; k, n-k \geq 2$.

This is similar to Case 1.2 (but easier because of the fact that $\left(\rho+\lambda, e_{1}+e_{j}\right)=$ $O\left(m_{k}\right)$ for all $\left.j\right)$. 
Type $D_{n}$

Case 4.1 Maximal subroot system is type $D_{n-1}$.

Assume $s \leq 1(n-1)$ and

$$
\Phi^{+\prime}=\left\{e_{i} \pm e_{j}: 1 \leq i<j \leq n ; i, j \neq n_{0}\right\}
$$

The case when $k \geq n_{0}$ can be done directly by counting, but is slightly different from the earlier cases because of the fact that $\left(e_{1}+e_{n}, \lambda_{n-1}\right)=0$. Observe that $\Phi^{+} \backslash \Phi^{+\prime}=\left\{e_{i} \pm e_{n_{0}}: i \neq n_{0}\right\}$ (where $e_{i}-e_{n_{0}}$ should be understood to mean $e_{n_{0}}-e_{i}$ when $\left.i>n_{0}\right)$. Because $k \geq n_{0}$,

$$
(\rho+\lambda, \alpha) \geq m_{k} \quad \text { for } \alpha= \begin{cases}e_{i}+e_{n_{0}} & \forall i \neq n_{0} \text {, provided } k \neq n-1 \\ e_{i}+e_{n_{0}} & \forall i \neq n_{0} \text { or } n, \text { if } k=n-1 \\ e_{n_{0}}-e_{n} & \text { if } \left.k=n-1 \text { (so that } n_{0} \neq n\right)\end{cases}
$$

Thus for all $k \geq n_{0},(\rho+\lambda, \alpha) \geq m_{k}$ for at least $n-1$ elements in $\Phi^{+} \backslash \Phi^{+\prime}$, and so

$$
\prod_{\alpha \in \Phi^{+} \backslash \Phi^{++}}(\rho+\lambda, \alpha)^{s-1} \leq m_{k}^{(n-1)(s-1)} .
$$

Combined with the fact that $\left|\Phi^{+\prime}\right|=2\left(\begin{array}{c}n-1 \\ 2\end{array}\right)$, this yields

$$
\prod_{\alpha \in \Phi^{+}}(\rho+\lambda, \alpha)^{s} \prod_{\alpha \in \Phi^{+} \backslash \Phi^{+}}(\rho+\lambda, \alpha)^{s-1} \leq c m_{k}^{s(n-1)(n-2)+(s-1)(n-1)},
$$

which is clearly bounded when $s \leq 1 /(n-1)$.

If $k=n-1$ and $n_{0}=n$, then $\left(\rho+\lambda, e_{i}-e_{n_{0}}\right)=O\left(m_{k}\right)$ for all $i=1, \ldots, n-1$ and so the argument is similar.

Otherwise we proceed inductively. The words from $\Phi^{+}$and $\Phi^{+\prime}$ based on the letters $\{2, \ldots, n\}$ are a subroot system of type $D_{n-2}$ in $D_{n-1}$ and so are handled by the inductive hypothesis, leaving us to show that

$$
\prod_{j \neq 1, n_{0}}\left(\rho+\lambda, e_{1} \pm e_{j}\right)^{s} \prod_{\alpha=e_{1} \pm e_{n_{0}}}(\rho+\lambda, \alpha)^{s-1}
$$

is bounded. But this is quite routine because the assumptions $k \leq n_{0}-1$ and $k \neq n-1$ ensure that $\left(\rho+\lambda, e_{1} \pm e_{n_{0}}\right) \geq m_{k}$.

Case 4.2 Maximal subroot system is type $A_{n-1}$.

It is convenient for the induction argument used in this case to assume $\Phi^{+\prime}=$ $\left\{s_{i} e_{i}-s_{j} e_{j}: i<j\right\}$, taking no consideration for the parity of the signs, $s_{i}$. We leave the initial case of $n=4$ for the reader, so assume $n>4$ and proceed inductively. 
Suppose first that $k \leq n-2$. Applying the induction argument one can see that it suffices to establish the boundedness of

$$
\left|\prod_{\alpha \in \Psi^{\prime}}(\rho+\lambda, \alpha)^{s} \prod_{\alpha \in \Psi \backslash \Psi^{\prime}}(\rho+\lambda, \alpha)^{s-1}\right|,
$$

where $\Psi=\left\{e_{1} \pm e_{j}: 1<j \leq n\right\}$ and $\Psi^{\prime}=\Psi \cap \Phi^{+\prime}$. Because $(\rho+\lambda, \alpha) \geq m_{k}$ for $\alpha=e_{1} \pm e_{n-1}$ and $\alpha=e_{1} \pm e_{n}$, at least two of which belong to $\Psi \backslash \Psi^{\prime}$, and $\left|\Psi^{\prime}\right|=n-1$, the product above is at most $\mathrm{cm}_{k}^{s(n-1)+2(s-1)}$ and hence is bounded when $s \leq 1 /(n-1)$.

If $k=n$, then let $J$ denote the number of $s_{j}=+1$. Notice that if $s_{i}$ and $s_{j}$ are the same sign, then $\left|\left(\rho+\lambda, s_{i} e_{i}+s_{j} e_{j}\right)\right| \geq m_{n}$. A counting argument shows that

$$
\left|\prod_{\alpha \in \Phi^{+}}(\rho+\lambda, \alpha)^{s} \prod_{\alpha \in \Phi^{+} \backslash \Phi^{+\prime}}(\rho+\lambda, \alpha)^{s-1}\right| \leq c m_{n}^{\left(\begin{array}{l}
n \\
2
\end{array}\right) s} m_{n}^{(s-1)\left(\left(\begin{array}{c}
j \\
2
\end{array}\right)+\left(\begin{array}{c}
n-j \\
2
\end{array}\right)\right)}
$$

and one can readily verify that this exponent is negative for our choices of $s$ and $n$.

The case $k=n-1$ is similar letting $J$ denote the number of elements of $\left\{s_{1}, \ldots, s_{n-1},-s_{n}\right\}$ equal to +1 .

Case 4.3 Maximal subroot system is type $D_{k} \times D_{n-k} ; k, n-k \geq 2$.

Here it is convenient for the induction argument to allow $k$ or $n-k$ to equal 1 , understanding that $D_{1}$ is the empty set. When $n=3$ we can only have $D_{1} \times D_{2}$, which is actually just $D_{2}$, and this was done in Case 4.1 of this section. (Indeed, Case 4.1 does $D_{1} \times D_{n-1}$ for general $n$.) This begins the induction argument.

From the previous remarks one can see there is no loss of generality in assuming $k$ and $n-k \geq 2$. Moreover, we may assume

$$
\Phi^{+\prime}=\left\{e_{i} \pm e_{j}: i<j ; i, j \in J_{1}\right\} \cup\left\{e_{i} \pm e_{j}: i<j ; i, j \in J_{2}\right\},
$$

where $J_{1}$ and $J_{2}$ are disjoint subsets of $\{1, \ldots, n\}$ of sizes $k$ and $n-k$, and $1 \in J_{1}$.

The induction argument applies to the factors with $\alpha=e_{i} \pm e_{j}, i, j \neq 1$, thus we need only consider the product over the remaining words:

$$
\prod_{\alpha \in\left\{e_{1} \pm e_{j}: i \neq j \in J_{1}\right\}}(\rho+\lambda, \alpha)^{s} \prod_{\alpha \in\left\{e_{1} \pm e_{j} ; j \in J_{2}\right\}}(\rho+\lambda, \alpha)^{s-1} .
$$

If $k \neq n-1$, then $\left(\rho+\lambda, e_{1}+e_{j}\right) \geq m_{k}$ for all $j \in J_{2}$. If $k=n-1$, it is still true that $\left(\rho+\lambda, e_{1}+e_{j}\right) \geq m_{k}$ for all $j \in J_{2}$ except $j=n$, but then also $\left(\rho+\lambda, e_{1}-e_{n}\right) \geq m_{k}$. In either case there are at least $\left|J_{2}\right|$ positive roots $\alpha \in\left\{e_{1} \pm e_{j}: j \in J_{2}\right\}$ such that $(\rho+\lambda, \alpha) \geq m_{k}$. As $\left|J_{2}\right| \geq 2$ and $\left|J_{1}\right| \leq n-2$, this implies (3.6) is bounded when $s \leq 1 /(n-1)$ and completes the proof for type $D_{n}$.

REMARK 3.1. The expressions obtained for the maximal subroot systems of the exceptional Lie groups, $E_{6}, E_{7}$, and $E_{8}$, are too cumbersome for the application of this method. 


\section{Optimality of the upper bounds}

In this section we demonstrate the optimality of the choice of $s$ in the main theorem, in the sense that there exist $g \in G$ and infinitely many representations $\lambda$ such that $\operatorname{Tr} \lambda(g)=O\left(d_{\lambda}^{1-s}\right)$. The elements $g$ in the torus $T$ which we work with, and the corresponding sets $\Phi^{+}(g)$, are listed below. Notice that the sets $\Phi^{+}(g)$ are the positive roots of maximal subroot subsystems of type $A_{n-1}, D_{n}, C_{1} \times C_{n-1}$ and $D_{n-1}$ in $A_{n}$, $B_{n}, C_{n}$ and $D_{n}$ respectively.

$\begin{array}{lll}\text { Type } & \text { Element } g \text { of } T & \text { Positive subroot system } \Phi^{+}(g) \\ A_{n} & (-n x, x, \ldots, x) \in \mathbb{R}^{n+1}, & \left\{e_{i}-e_{j}: 2 \leq i<j \leq n+1\right\} \\ & \text { where } x=\pi /(n+1) & \\ B_{n} & (\pi, \ldots, \pi) & \left\{e_{i} \pm e_{j}: 1 \leq i<j \leq n\right\} \\ C_{n} & (\pi, 0, \ldots, 0) & \left\{2 e_{1}\right\} \cup\left\{e_{i} \pm e_{j}, 2 e_{k}: i<j, k \neq 1\right\} \\ D_{n} & (\pi, 0, \ldots, 0) & \left\{e_{i} \pm e_{j}: 1<i<j \leq n\right\}\end{array}$

THEOREM 4.1. Suppose $G$ is a compact, connected, simple Lie group of type $A_{n}$, $B_{n}, C_{n}$ or $D_{n}$. Let $g$ be the element in $T$ listed in the chart above and let $\lambda=m \lambda_{1}$ with $m$ an even integer $\left(\lambda=m \lambda_{3}\right.$ in type $\left.C_{3}\right)$. Then

$$
\left|\frac{\operatorname{Tr} \lambda(g)}{\operatorname{deg} \lambda}\right| \geq \tilde{c}(g)(\operatorname{deg} \lambda)^{-s}
$$

for some constant $\tilde{c}(g)$ independent of $\lambda$ if

$$
s= \begin{cases}1 /(n-1) & \text { if } G \text { is type } A_{n-1} \text { or } D_{n} \\ 1 /(2 n-1) & \text { if } G \text { is type } B_{n} \\ 2 /(2 n-1) & \text { if } G \text { is type } C_{n}, n \neq 3 \\ 1 / 3 & \text { if } G \text { is type } C_{3} .\end{cases}
$$

The strategy of the proof is to first establish that

$$
\operatorname{det} w \operatorname{sgn}\left(\prod_{\alpha \in \Phi^{+}(g)}(\rho+\lambda, w(\alpha))\right) \exp i(\rho+\lambda, w(g))
$$

is constant over $w \in W$. This fact, together with (2.2), show that

$$
\left|\frac{\operatorname{Tr} \lambda(g)}{d_{\lambda}}\right| \geq \max _{w \in W} \tilde{c}(g) \frac{\left|\prod_{\alpha \in \Phi^{+}(g)}(\rho+\lambda, w(\alpha))\right|}{\prod_{\alpha \in \Phi^{+}}(\rho+\lambda, \alpha)},
$$

and we shall see that it is a straightforward matter to prove that the latter ratio is $O\left(d_{\lambda}^{-s}\right)$.

First, some preliminary results. 
LEMMA 4.2. Let $\lambda$ be any representation, let $\Phi^{+}(g)$ be as above and let $w=$ $w_{1} w_{2} \in W$ where $w_{1}$ is a product of sign changes and $w_{2}$ a permutation. $\left(w_{1}=1\right.$ in type $A_{n}$.) Then

$$
\operatorname{sgn}\left(\prod_{\alpha \in \Phi^{+}(g)}(\rho+\lambda, w(\alpha))\right)= \begin{cases}\operatorname{det} w_{2} & \text { in type } B_{n} ; \\ (-1)^{w_{2}(1)-1} \operatorname{det} w & \text { in type } A_{n}, C_{n} \text { or } D_{n} .\end{cases}
$$

ProOF. Obviously $\operatorname{sgn}\left(\left(\rho+\lambda, w_{2}(\alpha)\right)\right)=1$ when $\alpha=e_{i}+e_{j}, e_{i}$ or $2 e_{i}$. If $i<j$ and $w_{2}(i)<w_{2}(j)$, then $\operatorname{sgn}\left(\left(\rho+\lambda, w_{2}\left(e_{i}-e_{j}\right)\right)\right)=1$, while if $w_{2}$ reverses their order the sign is negative. Thus if we let

$$
X=\left\{(i, j): e_{i}-e_{j} \in \Phi^{+}(g), i<j \text { and } w_{2}(i)>w_{2}(j)\right\}
$$

then

$$
\operatorname{sgn}\left(\prod_{\alpha \in \Phi^{+}(g)}(\rho+\lambda, w(\alpha))\right)=(-1)^{|X|}
$$

In type $B_{n}$,

$$
X=\left\{(i, j): 1 \leq i<j \leq n \text { and } w_{2}(i)>w_{2}(j)\right\}
$$

hence $(-1)^{|X|}=\operatorname{det} w_{2}$. For the other types the pairs $(1, j)$ are never included in $X$ and therefore

$$
\operatorname{det} w_{2}=(-1)^{|X|+|| j: j>1 \text { and } w_{2}(1)>w_{2}(j)||}=(-1)^{|X|+w_{2}(1)-1} .
$$

Hence,

$$
(-1)^{|X|}=(-1)^{w_{2}(1)-1} \operatorname{det} w_{2}
$$

in types $A_{n}, C_{n}$ or $D_{n}$. This completes the proof for type $A_{n}$ as $w_{2}=w$.

Next, assume $w_{1}$ is a simple sign change, say $w_{1}\left(e_{i}\right)=-e_{i}$ if $i=i_{0}$ and $w_{1}\left(e_{i}\right)=e_{i}$ otherwise. Then

$$
\left(\rho+\lambda, w_{1}\left(e_{i_{0}}+e_{k}\right)\right)\left(\rho+\lambda, w_{1}\left(e_{i_{0}}-e_{k}\right)\right)=\left(\rho+\lambda, e_{i_{0}}+e_{k}\right)\left(\rho+\lambda, e_{i_{0}}-e_{k}\right),
$$

while of course $\left(\rho+\lambda, w_{1}\left(e_{i_{0}}\right)\right)=-\left(\rho+\lambda, e_{i_{0}}\right)$. Since $\Phi^{+}(g)$ only contains words of the form $e_{i} \pm e_{j}$ in types $B_{n}$ and $D_{n}$,

$$
\prod_{\alpha \in \Phi^{+}(g)}\left(\rho+\lambda, w_{1}(\alpha)\right)= \begin{cases}-\prod_{\alpha \in \Phi^{+}(g)}(\rho+\lambda, \alpha) & \text { in type } C_{n} ; \\ +\prod_{\alpha \in \Phi^{+}(g)}(\rho+\lambda, \alpha) & \text { in type } B_{n} \text { or } D_{n} .\end{cases}
$$


We can determine the effect of an arbitrary sign change by repeating this argument the appropriate number of times:

$$
\operatorname{sgn}\left(\prod_{\alpha \in \Phi^{+}(g)}\left(\rho+\lambda, w_{1}(\alpha)\right)\right)= \begin{cases}(-1)^{\# \text { sign changes }}=\operatorname{det} w_{1} & \text { in type } C_{n} ; \\ +1 & \text { in type } B_{n} \text { or } D_{n} .\end{cases}
$$

This is also the determinant of $w_{1}$ in type $D_{n}$ since only an even number of sign changes are allowed in the Weyl group.

Combining these observations completes the proof.

LEMMA 4.3. Let $g \in G$ be as above and let $\lambda=\sum m_{i} \lambda_{i}$ with $m_{i}$ even (and $m_{n} \equiv m_{n-1} \equiv 0 \bmod 4$ in type $\left.D_{n}\right)$. Let $w \in W, w=w_{1} w_{2}$, where $w_{1}$ is a product of sign changes $\left(w_{1}=1\right.$ in type $\left.A_{n}\right)$ and $w_{2}$ a permutation. Then

$$
\exp i(\rho+\lambda, w(g))= \begin{cases}(-1)^{w_{2}(1)-1} \theta & \text { in type } A_{n}, C_{n} \text { or } D_{n} ; \\ \operatorname{det} w_{1} \theta & \text { in type } B_{n}\end{cases}
$$

for some complex numbers $\theta$ of modulus one which do not depend on $w$.

PROOF. Type $B_{n}$. Here $w_{2}$ is clearly irrelevant. Expressed in terms of the standard basis vectors the $j^{\prime}$ th entry of $\rho+\sum m_{i} \lambda_{i}$ is

$$
\begin{cases}\sum_{i=j}^{n-1} m_{i}+m_{n} / 2+n-j+1 / 2 & \text { if } j \neq n ; \\ \left(m_{n}+1\right) / 2 & \text { if } j=n .\end{cases}
$$

The reader can easily check from this that if $w_{1}$ changes $k$ signs, then

$$
\exp i(\rho+\lambda, w(g))=(-1)^{k} \theta=\operatorname{det} w_{1} \theta
$$

for an appropriate choice of $\theta$.

Type $C_{n}$. In terms of the standard basis vectors

$$
\rho+\sum m_{i} \lambda_{i}=\left(\sum_{i=1}^{n} m_{i}+n, \sum_{i=2}^{n} m_{i}+n-1, \ldots, m_{n}+1\right) .
$$

Suppose $w_{2}(1)=j$. Then,

$$
\left(\rho+\lambda, w_{2}(g)\right)=\left(\sum_{i=j}^{n} m_{i}+n-j+1\right) \pi .
$$

As all $m_{i}$ are assumed even,

$$
\exp i\left(\rho+\lambda, w_{2}(g)\right)=(-1)^{j-1} \exp i n \pi .
$$


Because $g=-g$, the sign changes have no effect on $g$ and thus the argument is complete.

Type $D_{n}$. One can verify that if $w_{2}(1)=j$, then

$$
(\rho+\lambda, w(g))= \begin{cases} \pm\left(\sum_{i=j}^{n-2} m_{i}+\left(m_{n-1}+m_{n}\right) / 2+n-j\right) \pi & \text { if } j \leq n-2 \\ \pm\left(1+\left(m_{n-1}+m_{n}\right) / 2\right) \pi & \text { if } j=n-1 \\ \pm\left(m_{n}-m_{n-1}\right) \pi / 2 & \text { if } j=n\end{cases}
$$

with the choice of \pm depending on $w_{1}$. As these are all integer multiples of $\pi$ the choice of \pm does not affect the parity of $(\rho+\lambda, w(g))$, and since $m_{1}, \ldots, m_{n-2}$ and $1 / 2\left(m_{n-1} \pm m_{n}\right)$ are even integers, it follows that for all choices of $j$ we have

$$
\exp i(\rho+\lambda, w(g))=(-1)^{j} \exp i n \pi .
$$

Type $A_{n}$. The $j^{\prime}$ th entry of $\rho+\sum m_{i} \lambda_{i}$ is

$$
\frac{1}{n+1}\left(-\left(m_{1}+1\right)-2\left(m_{2}+1\right)+\cdots+\left(m_{j}+1\right)(n-j+1)+\cdots+\left(m_{n}+1\right)\right) \text {. }
$$

The same kinds of calculations as used for the other types show that if $w_{2}(1)=j$ then

$$
\exp i(\rho+\lambda, w(g))=\exp i(\rho+\lambda, g)(-1)^{j-1} .
$$

PROOF OF THEOREM 4.1. Combining these lemmas we clearly obtain

$$
\operatorname{det} w \operatorname{sgn}\left(\prod_{\alpha \in \Phi^{+}(g)}(\rho+\lambda, w(\alpha))\right) \exp i(\rho+\lambda, w(g))=\theta
$$

and this is independent of $w$. Thus

$$
\frac{|\operatorname{Tr} \lambda(g)|}{d_{\lambda}} \geq \tilde{c}(g) \frac{\max _{w \in W}\left|\prod_{\alpha \in \Phi^{+}(g)}(\rho+\lambda, w(\alpha))\right|}{\prod_{\alpha \in \Phi^{+}}(\rho+\lambda, \alpha)} .
$$

For type $B_{n}$ notice that $\Phi^{+}(g) \supseteq\left\{e_{1} \pm e_{j}: j \neq 1\right\}$. As $\lambda=m \lambda_{1}$ we have $\left(\rho+\lambda, e_{1} \pm e_{j}\right) \geq m$, thus

$$
\max _{w \in W}\left|\prod_{\alpha \in \Phi^{+}(g)}(\rho+\lambda, w(\alpha))\right| \geq c m^{2(n-1)} .
$$

Since also $\left(\rho+\lambda, e_{\mathrm{r}}\right)=O(m)$ and $(\rho+\lambda, \alpha)$ is bounded independently of $m$ for all other $\alpha \in \Phi^{+}$, it follows that $d_{\lambda}=c m^{2 n-1}$ and hence

$$
\frac{|\operatorname{Tr} \lambda(g)|}{d_{\lambda}} \geq c d_{\lambda}^{-1 /(2 n-1)}
$$

as claimed.

The other cases are similar. 


\section{Singularity of central, continuous measures}

A measure $\mu$ on $G$ is called central if $\mu$ commutes with all other measures on $G$ under the action of convolution. Central measures are characterized by the fact that their Fourier transforms are scalar multiples of identity matrices:

$$
\widehat{\mu}(\lambda)=a_{\lambda} I_{d_{\lambda}}, \quad \text { where } a_{\lambda}=\int_{G} \frac{\operatorname{Tr} \lambda(x)}{d_{\lambda}} d \mu .
$$

We simply write $\widehat{\mu}(\lambda)$ in place of $a_{\lambda}$.

An interesting class of singular, central measures are the orbital measures. The orbital measure $\mu_{g}$, supported on the conjugacy class $C(g)$ containing $g \in G$, is defined by

$$
\int_{G} f d \mu_{g}=\int_{G} f\left(t g t^{-1}\right) d m_{G}(t) \quad \text { for } f \in C(G) .
$$

Orbital measures are continuous if and only if $g \notin Z(G)$, the centre of $G$.

In [8] Ragozin proved that if $g \notin Z(G)$, then $\mu_{g}^{\operatorname{dim} G} \in L^{1}(G)$. One can easily see that $\widehat{\mu_{g}}(\lambda)=\operatorname{Tr} \lambda(g) / d_{\lambda}$, and using this fact it was shown in [2] that if $k>\operatorname{dim} G / 2$, then $\mu_{g}^{k} \in L^{2}$. By appealing to the sharper results of this paper we can now prove:

PROPOSITION 5.1. The measures $\mu_{g}^{k}$ belong to $L^{2}(G)$ for all $g \notin Z(G)$ if and only if $k \geq k_{0}$, where

$$
k_{0}= \begin{cases}n & \text { if } G \text { is type } A_{n-1} ; C_{n}, n \neq 3 ; \text { or } D_{n} \\ 2 n & \text { if } G \text { is type } B_{n} \\ 4 & \text { if } G \text { is type } C_{3} .\end{cases}
$$

PROOF. From the Peter-Weyl theorem we know $\mu_{g}^{k} \in L^{2}$ if and only if

$$
\sum_{\lambda \in \widehat{G}} d_{\lambda}\left|\widehat{\mu}_{g}(\lambda)\right|^{2 k} \operatorname{Tr}\left|l_{d_{\lambda}}\right|^{2}=\sum_{\lambda \in \widehat{G}} d_{\lambda}^{2}\left|\frac{\operatorname{Tr} \lambda(g)}{d_{\lambda}}\right|^{2 k}<\infty .
$$

It was shown in Corollary 9 of [2] that $\sum_{\lambda \in \widehat{G}} d_{\lambda}^{t}<\infty$ when $t<-\operatorname{rank} G /\left|\Phi^{+}\right|$. This fact, combined with Theorem 3.1, proves the sufficiency of the choice of $k$.

Necessity is a consequence of Theorem 4.1. For example, when $G$ is type $A_{n}$ and $g=(-n x, x, \ldots, x)$ for $x=\pi /(n+1)$ we know

$$
\begin{aligned}
\sum_{\lambda \in \widehat{G}} d_{\lambda}^{2}\left|\frac{\operatorname{Tr} \lambda(g)}{d_{\lambda}}\right|^{2 k} & \geq \sum_{m \text { even }} d_{m \lambda_{1}}^{2}\left|\frac{\operatorname{Tr} m \lambda_{1}(g)}{d_{m \lambda_{1}}}\right|^{2 k} \\
& \geq \tilde{c}(g) \sum_{m \text { even }} d_{m \lambda_{1}}^{2} d_{m \lambda_{1}}^{-2 k / n}=c \sum_{m \text { even }} m^{n(2-2 k / n)},
\end{aligned}
$$


which is finite only if $2 n-2 k<-1$. Thus we require $k>n+1 / 2$, but as $k \in \mathbb{N}$ this means $k \geq n+1$ is a necessary condition. The other types are similar.

REMARK 5.1. Of course, if $\mu_{g}^{k} \in L^{2}$ then $\mu_{g}^{2 k}$ is a continuous function.

The same result can be proved for central, continuous measures compactly supported on the conjugates of a set of the form $\left\{x \in T: \Phi^{+}(x)=\Phi^{+\prime}\right\}$ for some fixed set $\Phi^{+\prime}$ as such measures $\mu$ also have the property that $|\widehat{\mu}(\lambda)| \leq O\left(d_{\lambda}^{-s}\right)$ for $s$ as in the main theorem (see [2]). We should point out, in contrast, that for any $a<1$ there are central, continuous measures $\mu$ such that $\widehat{\mu}(\lambda) \geq d_{\lambda}^{a-1}$ for infinitely many $\lambda$. This is shown in [3] and is a consequence of the fact that although compact Lie groups do not admit infinite central Sidon sets (an application of Ragozin's original work) they do admit central $(a, 1)$-Sidon sets for all $a<1$.

Finally, we are ready to improve upon Ragozin's result on convolutions of arbitrary central, continuous measures.

PROPOSITION 5.2. Suppose $\mu_{1}, \ldots, \mu_{k}$ are central continuous measures and $k \geq$ $k_{0}$. Then $\mu_{1} * \cdots * \mu_{k} \in L^{1}(G)$.

PROOF. The proof is essentially the same as Theorem 11 of [2] but uses the stronger results obtained in Proposition 5.1.

REMARK 5.2. Ragozin observed that $\mu_{g}^{k}$ is singular to Haar measure on $G$ for all $k<\operatorname{dim} G / \operatorname{dim} C(g) . A s \operatorname{dim} C(g)=2\left(\left|\Phi^{+}\right|-\left|\Phi^{+}(g)\right|\right)$ ([7]) this means, for instance, that if $G$ is type $A_{n}$, then $\mu_{g}^{k}$ is singular to Haar measure when $k<n / 2+1$. It remains open as to whether or not $\mu_{g}^{k} \in L^{1}$ for all $g \in G \backslash Z(G)$, when $k$ is between $n / 2+1$ and $n+1$ (other than for the trivial case $A_{1}$, where clearly $k=2$ is the best possible result).

A measure $\mu$ is called $L^{p}$-improving if there is some $p<2$ such that $\mu * L^{p} \subseteq L^{2}$. Young's inequality implies that all functions in $L^{q}$, for some $q>1$, are examples of $L^{p}$-improving measures. A question of current interest is to understand which singular measures on compact groups are $L^{p}$-improving. For example, surface measures on analytic manifolds which generate $G$ were shown to be $L^{p}$-improving in [9]. In [10] it was shown that if $g$ was a regular element, then $\mu_{g} * L^{p} \subseteq L^{2}$ if and only if $p \geq 1+r /(2 \operatorname{dim} G-r)$. For arbitrary continuous, orbital measures we can prove:

Proposition 5.3. If $g \notin Z(G)$, then $\mu_{g}$ is $L^{p}$-improving. Indeed, for any $g \notin$ $Z(G), \mu_{g} * L^{p} \subseteq L^{2}$ for $p>2-2 /(n+1)$ when $G$ is type $A_{n-1}, D_{n}$ or $C_{n}, n \neq 3$; $p>2-2 /(2 n+1)$ in type $B_{n}$; and $p>8 / 5$ for $C_{3}$. 
Proof. Proposition 5.1 tells us that the operator $T_{k_{0}}(f)=\mu_{g}^{k_{0}} * f$ maps $L^{1}(G)$ into $L^{2}(G)$ whenever $g \notin Z(G)$. Since the identity map obviously maps $L^{2}(G)$ into $L^{2}(G)$ an application of Stein's interpolation theorem [12] (see also [4]) gives that $\mu_{g} * L^{p} \subseteq L^{2}$ for the choices of $p$ listed.

\section{References}

[1] T. Brocker and T. Dieck, Representations of compact Lie groups (Springer, New York, 1985).

[2] K. Hare, 'The size of characters of compact Lie groups', Studia Math. 129 (1998), 1-18.

[3] — , 'Central Sidonicity for compact Lie groups', Ann. Inst. Fourier (Grenoble) 45 (1995), $547-564$.

[4] - 'Properties and examples of $\left(L^{p}, L^{q}\right)$ multipliers', Indiana Univ. Math. J. 338 (1989), 211-227.

[5] J. Humphreys, Introduction to Lie algebras and representation theory (Springer, New York, 1972).

[6] W. McKay, J. Patera and D. Rand, Tables of representations of simple Lie algebras, vol. 1 Exceptional simple Lie algebras, Centre de Recherches Math., Univ. of Montreal, 1990.

[7] M. Mimura and H. Toda, Topology of Lie groups, Transl. Math. Monographs 91 (Amer. Math. Soc., Providence, R.I., 1991).

[8] D. Ragozin, 'Central measures on compact simple Lie groups', J. Funct. Anal. 10 (1972), 212-229.

[9] F. Ricci and E. M. Stein, 'Harmonic analysis on' nilpotent groups and singular integrals. III. Fractional integration along manifolds', J. Funct. Anal. 86 (1989), 360-389.

[10] F. Ricci and G. Travaglini, ' $L^{p}-L^{q}$ estimates for orbital measures and Radon transform on compact Lie groups and Lie algebras', J. Funct. Anal. 129 (1995), 132-147.

[11] D. Rider, 'Central lacunary sets', Monatsh. Math. 76 (1972), 328-338.

[12] E. M. Stein and G. Weiss, Introduction to Fourier analysis on Euclidean spaces (Princeton Univ. Press, Princeton, 1971).

[13] V. Varadarajan, Lie groups, Lie algebras and their representations (Springer, New York, 1984).

Department of Pure Mathematics

PO Box 280

University of Waterloo

Churchill VIC 3842

Waterloo, Ont.

Australia

Canada

e-mail: davidw@utopiatype.com.au

e-mail: kehare@uwaterloo.ca 